\title{
Integrating an Electronic Health Record System into a Regional Health Information System: An HL7 FHIR Architecture
}

\author{
Elena LAZAROVA ${ }^{\text {a }}$, Sara MORA ${ }^{\mathrm{a}}$, Paolo RUBARTELLI ${ }^{\mathrm{b}}$, \\ Alessandro COSOLITO VITALE ${ }^{\mathrm{b}}$, Luisa PARETO ${ }^{\mathrm{b}}$, Norbert MAGGI ${ }^{\mathrm{a}, \mathrm{c}}$, \\ Carmelina RUGGIERO ${ }^{\mathrm{a}}$ and Mauro GIACOMINI ${ }^{\mathrm{a}, 1}$ \\ ${ }^{a}$ Department of Informatics, Bioengineering, Robotics and System Engineering \\ (DIBRIS), University of Genoa, Italy \\ ${ }^{\mathrm{b}}$ Local Health Authority of Genoa (ASL3), Genoa, Italy \\ 'IRCCS Istituto G. Gaslini, Genoa, Italy
}

\begin{abstract}
This paper presents an architecture which has been developed in order to integrate a routinely used cardiologic EHR system into the health information infrastructure system of the region where the EHR is used. A Service Oriented Approach based on HL7 and FHIR was used, achieving interoperability, security and confidentiality at all levels. The system has been used for about a year and has brought about significant improvements in the provision of care.
\end{abstract}

Keywords. Electronic Health Record, Health Information Infrastructure, HL7

\section{Introduction}

Electronic Health Records (EHR) have been increasingly used in the past decade in healthcare units and individual organisations on a regional level or nationwide. Their implementation in hospitals is particularly complex for several reasons, including the complexity of medical data, security and confidentiality concerns and data entry problems [1]. The adoption of EHRs automates data management, simplifies the clinician's workflow and supports care related activities, integrating data for different purposes, research and administrative aspects. The foreseeable widespread adoption of EHRs can improve quality, safety and efficiency in healthcare, even though several challenges should still be overcome. Enhanced standardisation can bring about significant improvements in healthcare [2]. To support the development of interoperable and reliable medical systems, several medical standards are being used, such as HL7, FHIR and others. HL7 mainly focuses on patient management [3] and introduced FHIR, which extended previous HL7 specifications by modern web technologies. The basic building blocks of FHIR are generic definitions of common healthcare concepts which are intended to cover typical use cases [4]. FHIR is being increasingly adopted and is regarded as an emerging standard for EHRs. The work presented here relates to the

\footnotetext{
${ }^{1}$ Corresponding Author, Mauro GIACOMINI; E-mail: mauro.giacomini@dibris.unige.it.
} 
integration among data obtained in hospitals (Villa Scassi) and to their integration into the regional health information system (Liguria region, Italy).

\section{Materials and Methods}

In Italy at national level most of the existing Health Information Infrastructure (HII) systems have adopted an institution-centred data storage, leaving patient records where they were created. Since the records are not centrally stored at least one central index is needed, by which the information of each patient can be extracted. When a patient data is requested the index is used to query the repository where the stored information is located. The requested data is then assembled obtaining the complete patient record. An architecture was developed to integrate a routinely used EHR system into the HII system. Specifically, a Service Oriented Approach based on HL7 and FHIR was used, achieving interoperability, security and confidentiality at all levels [5, 6]. The Logical Observation Identifier Names and Code (LOINC) was used [7].

\section{Results}

The current level of efficacy of cardiology allows to rapidly improve the conditions of most patients and to allow for their discharge. The clinical management of patients after hospitalization is also important to keep the patient in better health, preventing exacerbations and the need for new hospitalizations. The EHR system initially developed has been integrated in several respects, considering specific requirements such as the user identification, the request of reports, the patient admission registration, the Hospital Discharge Letter (HDL), and the access to radiology reports from EHR.

\section{Conclusions}

The system has been used in the Cardiology Department since December 2019 and has brought significant improvements in the provision of care. The patients' follow-up of by physicians after the discharge is supported to a greater extent by the ready availability of a detailed description of the therapies that have been administered in the hospital.

\section{References}

[1] Boonstra A, et al. Implementing electronic health records in hospitals: a systematic literature review. BMC Health Serv. Res. 2014;14(1): 370.

[2] Rahman R, Reddy CK. Electronic Health Records: A Survey. Healthc. Data Anal. 2015;36:21.

[3] Joyia GJ, et al., Evolution of health level-7: A survey, in Proceedings of the 2018 International Conference on Software Engineering and Information Management, 2018, pp. 118-123.

[4] Lehne M, et al. The Use of FHIR in Digital Health-A Review of the Scientific Literature. GMDS, 52-58, 2019.

[5] Gazzarata M, et al. A SOA-Based Platform to Support Clinical Data Sharing. J. Healthc. Eng. 2017.

[6] Gazzarata G, Gazzarata R, Giacomini M. A standardized SOA based solution to guarantee the secure access to HER. Procedia Comput. Sci. 2015;64:1124-1129.

[7] Bodenreider O, Cornet R, Vreeman DJ, Recent developments in clinical terminologies - SNOMED CT, LOINC, and RxNorm. Yearb. Med. Inform. 2018;27(1);129. 